\title{
Stable carbon monosulfide nanostructures: Chain arrays and monolayers
}

\author{
T. Alonso-Lanza, ${ }^{1}$ F. Aguilera-Granja, ${ }^{1,2}$ J. W. González, ${ }^{1}$ and A. Ayuela ${ }^{1}$ \\ ${ }^{1}$ Centro de Física de Materiales CFM-MPC CSIC-UPV/EHU, Donostia International Physics Center (DIPC), \\ Departamento de Física de Materiales, Fac. de Químicas, UPV-EHU, 20018 San Sebastián, Spain \\ ${ }^{2}$ Instituto de Física, Universidad Autónoma de San Luis de Potosí, 78000 San Luis Potosí S.L.P., México
}

(Received 12 April 2017; revised manuscript received 3 June 2017; published 12 July 2017)

\begin{abstract}
Herein we show using theoretical predictions that carbon monosulfide compounds exhibit a variety of layered nanostructures, such as chain arrays, monolayers, and thin films. We show that semiconductor chain arrays are the most stable because they are mainly dimensionality driven by $s p^{2}$ hybridization of the carbon orbitals. In contrast to the thin films, the monolayers are stable at room temperature in a semiconductor phase, which is followed in energy by a metallic phase. Moreover, we study a semiconductor-to-metal phase transition in the carbon monosulfide monolayers by strain engineering to control the conductivity and carrier mobility.
\end{abstract}

DOI: 10.1103/PhysRevMaterials.1.024001

\section{INTRODUCTION}

New phenomena in condensed matter physics are based on two-dimensional (2D) materials. One such example is graphene, which comprises single layers of carbon atoms [1]. Graphene exhibits outstanding structural and electronic properties such as the ability to have a high stiffness or flexibility, and its massless Dirac fermions with low resistivity [2-7], which enable many applications. The main drawbacks of graphene are the lack of a band gap in its electronic structure and its small on/off ratio [4,8]. A band gap may be opened via different techniques, such as chemical tailoring, inducing strain, and geometric patterning [9-14]. Although graphene looks promising for the future, current research has focused on other carbon nanostructured materials and other two-dimensional materials [15].

The ability to exfoliate materials and the existence of accurate experimental techniques to study single-layer materials [16-18] have inspired researchers to study other layered materials. Layers using other elements in the carbon group have been studied to produce graphene counterparts, such as silicene, germanene, and stanene [19-22]. Graphene is flat due to its $s p^{2}$ hybridization, and the other compounds adopt a buckled structure because they prefer $s p^{3}$ hybridization. Other layered nanostructures that are isoelectronic to graphene can be obtained by combining III-V group elements, such as hexagonal boron nitride [23]. 2D materials research has recently incorporated other new materials based on phosphorus, whose isoelectronic counterparts are a current research focus [24-26]. Black phosphorus is formed by single layers that are mediated by van der Waals interactions. Hence, analogous to graphene, a few layers can be isolated to form phosphorene [27,28]. Phosphorene has a two-dimensional honeycomb puckered structure in which each atom is bonded to three neighbors and displays a direct band gap that is tunable according to the number of layers and the degree of stacking [29,30]. The number of systems being proposed and comprehensively analyzed to obtain 2D materials with an improved stability and new properties is increasing [31]. The isoelectronic compounds of phosphorene are a hot topic of research.

Two different approaches exist for obtaining isoelectronic compounds of phosphorene: the use of elements from group $\mathrm{V}$ and by the mixing of elements from groups IV-VI, which are also referred to collectively as the group IV monochalcogenides [32-37]. The isoelectronic compounds of phosphorene [38] from group $\mathrm{V}$ are arsenene [39-41], antimonene [41] and their binary compounds $[42,43]$. These compounds are currently being investigated by studying their doping [44], point defects $[44,45]$, and oxygen contamination [46]. The group IV monochalcogenides include combinations of light elements, such as those in silicon monosulfide monolayers and thin films [24-26], and a combination of heavy (Ge, Sn) and light $(\mathrm{O}, \mathrm{S})$ atoms [47], such as in silicon telluride [48] and germanium monosulfide [49] monolayers, which exhibit strain-tunable indirect band gaps. Nevertheless, none of the currently proposed isoelectronic compounds of phosphorene are based on carbon. In fact, layered carbon compounds, such as carbon nitride [50] and carbon phosphide [51,52], are not isoelectronic to phosphorene. Our aim is to design new layered nanostructures that are isoelectronic to black phosphorus, but still composed of carbon.

Here we present stable carbon monosulfide nanostructures that cover chains, monolayers, and thin films. We perform phonon and molecular dynamics calculations to ensure the stability of these structures at room temperature. The carbon monosulfide nanostructures vary from having metallic properties in thin films to having semiconductor properties in chains and most monolayers. The chains and thin films are more stable than the monolayers because sulfur atoms have two bonds, instead of three (or four), in the monolayers [26]. The large variety of nanostructures that we explored with either metallic or semiconductor characteristics makes this material an interesting compound for several applications based on its electronic transport ability within semiconductors devices.

\section{COMPUTATIONAL DETAILS}

We used the SIESTA method to carry out density functional theory (DFT) calculations for carbon monosulfide nanostructures. To determine the exchange and correlation potentials, we used the Perdew-Burke-Ernzenhof form of the generalized gradient approximation (GGA) [53]. We fully relaxed the atomic positions and unit cells until the forces were below $0.006 \mathrm{eV} / \AA$. The used unit cells had large vectors of approximately $24.5 \AA$ perpendicular to the layers in order 


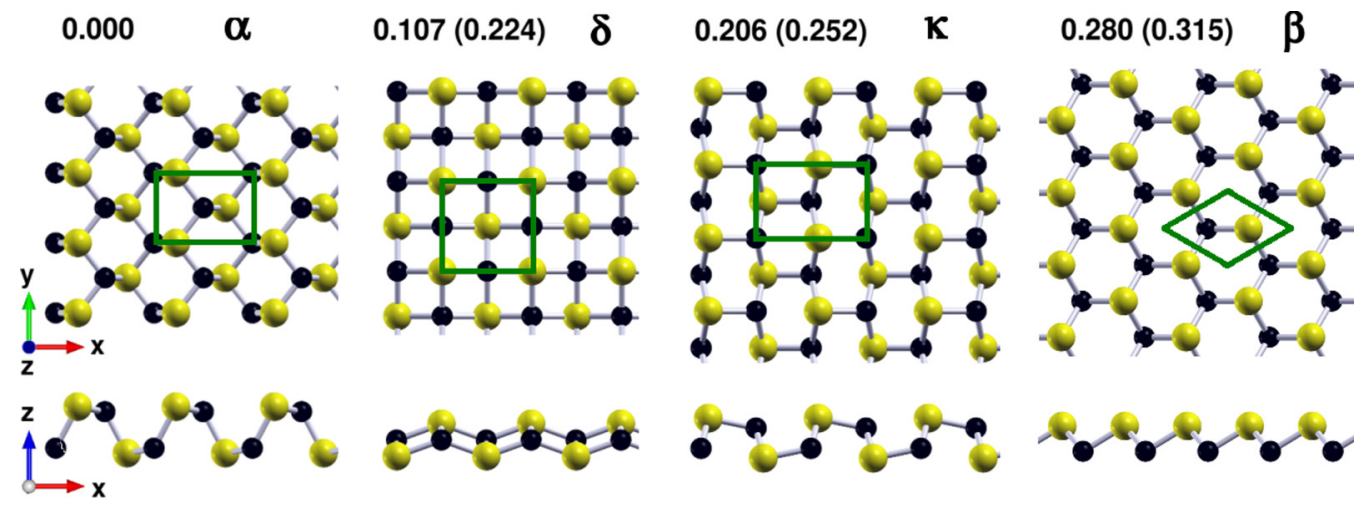

FIG. 1. Geometries of the four carbon monosulfide monolayers in order of decreasing stability. Carbon (sulfur) atoms are represented by black (yellow) spheres through the paper. The unit cell is shown in green for each case. Differences in the energy per atom using the SIESTA and VASP methods are shown on top and in parentheses for each structure, respectively. The energy differences are calculated relative to the energy per atom in the $\alpha$ structure.

to avoid interacting with images. An electronic temperature of $25 \mathrm{meV}$ and a mesh cutoff of $250 \mathrm{Ry}$ were used. The Brillouin zone was sampled with a fine grid of $20 \times 20 \times 1 k$ points. The atomic cores were described by nonlocal normconserving Troullier-Martins [54] pseudopotentials factorized in the Kleynman-Bylander form. The orbitals were developed using basis sets for each atom, and the basis size was double that of zeta size plus the polarization orbitals. Details on the used pseudopotentials and basis sets were described previously $[9,26,55]$. Our main results were checked by repeating the calculations with the VASP method using the projected augmented wave method (PAW) within the PBE formalism for the exchange and correlation effects [56,57], and the QUANTUM ESPRESSO method with equivalent parameters [58]. We estimated the van der Waals contributions to the total energy for a two-dimensional array with well-separated chains by using the implementation of the vdW-DF [59] functional within the VASP method $[60,61]$. Hybrid functional calculations were also carried out using plane wave methods. We confirmed the stability of the highly stable resulting structures by conducting molecular dynamics calculations within the SIESTA method using the Nose thermostat at room temperature. We employed a Nose mass [62-64] of $10 \mathrm{Ry} \mathrm{fs}{ }^{2}$, and a time step of $1 \mathrm{fs}$. We chose 3000 as the final time step and the relaxation time to reach the target temperature was 2500.0 fs. Phonon dispersion curves were obtained using the QUANTUM ESPRESSO method, and the details are included in the Supplemental Material [65].

\section{RESULTS AND DISCUSSION}

We study three different types of carbon monosulfide nanostructures in the following order (from low to high energy stability): monolayers, thin films, and chain arrays. Molecular dynamics simulations indicate that thin films are not stable at room temperature. Further comments and analyses of thin film structures are presented in the Supplemental Material [65], together with further information on the monolayers and chains.

\section{A. Monolayers}

We explore a wide variety of structures for the carbon monosulfide monolayers. Next, we present the geometries and electronic structures of the four most stable structures, and finally consider the ground state in more detail. Figure 1 shows four monolayers of carbon monosulfide after structural relaxation, including their relative energies. The most stable $\alpha$ monolayer presents a structure similar to that of a single layer of black phosphorus. This structure has also been reported recently for group IV monochalcogenides [24,32], and group $\mathrm{V}$ semiconductors such as phosphorous-nitrogen and arsenic-nitrogen $[42,43,66]$. The results obtained using the VASP method yielded slightly larger energy differences than those obtained with SIESTA, although the stability order is the same. We found that the $\delta$ monolayer has the second highest energy with a regular structure composed of squares when viewed from above. The side view reveals two different heights for the sulfur atoms. In addition, there is a similar structure, the $\kappa$ structure, in which all the carbon atoms are shifted toward one side, producing long and short distances between the sulfur atoms. When the distortion is maximized, the $\alpha$ structure is attained. The $\kappa$ layer appears to have structural similarities with the $\alpha$ and $\delta$ monolayers. Therefore, the three monolayer carbon monosulfide structures appears to be related, which is discussed at length in Sec. III A 2. The phonon dispersions, shown in the Supplemental Material [65], indicate that the $\alpha$ and $\delta$ structures are very stable, and the $\kappa$ structure is unstable due to the presence of negative frequencies. Furthermore, the molecular dynamics simulations show that the $\alpha$ and $\delta$ structures are stable even at room temperature. Lastly, we find the $\beta$ structure which displays a hexagonal pattern when viewed from above and a buckled structure when viewed from the side, as being reported for other isoelectronic compounds of phosphorene [24]. The $\beta$ structure is similar to that of blue phosphorene $[67,68]$. The phonon dispersion curves show that the carbon monosulfide $\beta$ phase is unstable.

To gain insight into the electronic structure of the carbon monosulfide monolayers we considered the band structures shown in Fig. 2. On the one hand, three of the monolayers, labeled as $\alpha, \kappa$, and $\beta$, are semiconductors with indirect band gaps of $0.97,0.77$, and $1.64 \mathrm{eV}$, respectively. For the $\alpha$ and $\kappa$ layers, the conduction band minimums are located at the gamma point. We define the eigenvalue gap as an energy gap between valence bands just below the Fermi energy. For the $\alpha$ 

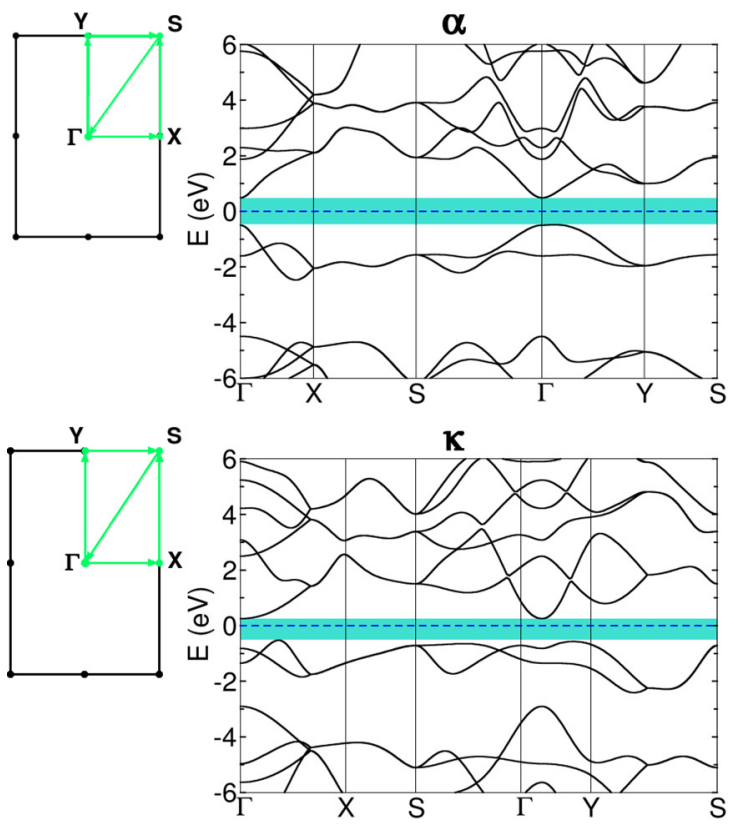
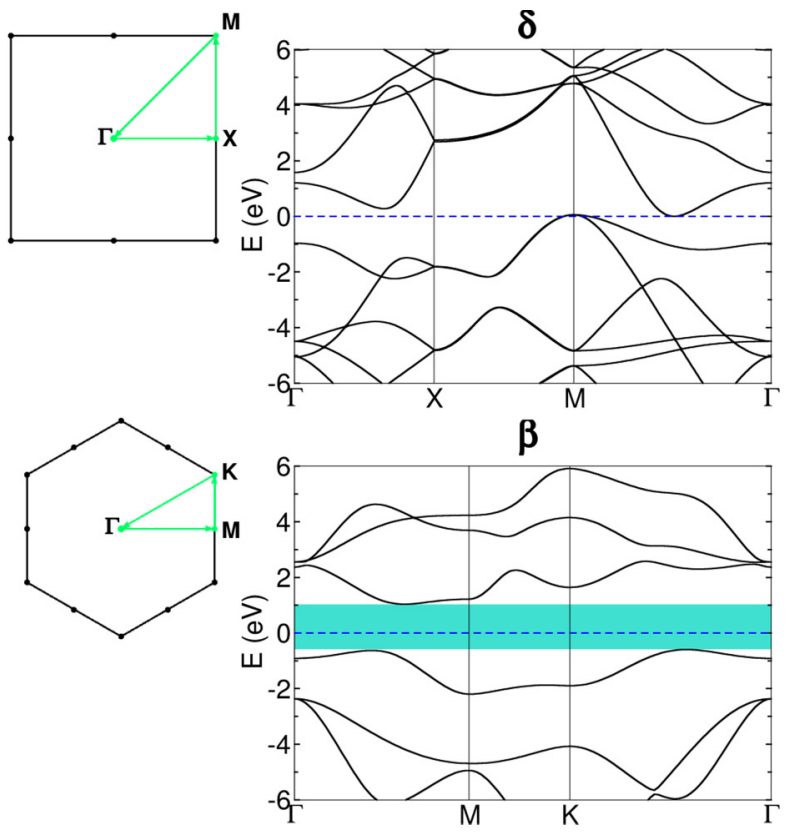

FIG. 2. Band structures of the four carbon monosulfide monolayers. Fermi energies are denoted by horizontal dashed lines. Band gaps over the whole Brillouin zone are shown by shaded rectangles.

case, the highest occupied valence band is distinctly separated from the lower valence bands with an eigenvalue gap of $2 \mathrm{eV}$. The $\kappa$ structure also has an eigenvalue gap in the valence band, although the value is reduced to $0.5 \mathrm{eV}$. On the other hand, the $\delta$ structure is metallic.

The bands of the $\alpha, \delta$, and $\kappa$ structures appear similar because they share common structural features, as mentioned above. For the $\beta$ monolayer the bands are degenerated throughout the Brillouin zone due to the lattice symmetry. Most of the features of the bands are the same for the $\alpha$ and $\kappa$ cases; for instance there are two occupied bands just below the Fermi energy, extended throughout the Brillouin zone, and distinctly separated from the deeper states. However, the band structure of the $\delta$ case differs because one of the two occupied bands near the Fermi energy becomes broad, and decreases to deep energies following a parabolic shape, destroying the eigenvalue gap. The two occupied narrow bands for the $\alpha$ and $\kappa$ cases are localized within a interval of $2 \mathrm{eV}$, while the bands for the $\delta$ case become broad and expand over more than $6 \mathrm{eV}$. The lowest energy conduction bands also become broad, similar to the valence bands near the Fermi energy. In addition, there are bands in the $\delta$ structure that cross the Fermi energy, resulting in a conducting monolayer. Because of the reported underestimation of the band gap when carrying out DFT-GGA calculations, we have carried out additional simulations using HSE hybrid functionals design for metallic systems. The results still supports the $\delta$ monolayer as metallic. Details are included in the Supplemental Material [65].

Furthermore, we studied the charge transfer between the carbon and sulfur atoms using the Mulliken scheme. The charge values are $0.81,0.92,0.65$, and 0.66 electrons for the $\alpha, \delta, \kappa$, and $\beta$ monolayers, respectively. In some cases, the charge transfer is sufficiently high to consider that one electron transfer from the carbon to the sulfur atoms. This result is surprising because carbon and sulfur have approximately the same Pauling electronegativity. Hence, the larger size of the sulfur atoms with respect to the carbon atoms must be taken into account in order to explain the charge gained by the sulfur atoms [69].

\section{1. $\alpha$ monolayer}

In this section we focus on the ground state monolayer induced by the $\alpha$ structure. Each carbon atom is bonded to three sulfur atoms and vice versa. There are two bonds with a length of $1.82 \AA$ and one bond with a length of $1.90 \AA$. The angle between the bonds from the carbon atom is $113^{\circ}$ in two cases and $103^{\circ}$ in the other case. The carbon atoms present $s p^{3}$-like hybridization.

Next, we discuss the relative contributions of the carbon and sulfur atoms in terms of stability. The projected density of states is shown in Fig. 3(a). The total states projected on sulfur contribute more than those on carbon because sulfur has a larger number of electrons than carbon. Nevertheless, the occupied peak near the Fermi energy has a larger carbon contribution than sulfur. This particular peak is related to the isolated valence band, as discussed above for Fig. 2. The density of states (DOS) in real space within the energy range of $2 \mathrm{eV}$ below the Fermi energy is included in Fig. 3(b). Most of the states are localized around the carbon atoms with orbital lobes that suggest large $p$-type contributions. The LDOS peak globally appears as a dangling bond of the $s p^{3}$ hybridization. However, the wave function for the highest occupied orbital at the $\Gamma$ point, as shown in Fig. 3(c), reveals a $p_{z}$-like orbital over the carbon atoms, which is attributed to the broadening of the band near the Fermi energy due to structural distortions. The projected sulfur LDOS shown in Fig. 3(a) has a non-negligible $d$ contribution, especially for the highest energy occupied peak. Therefore, $s p d$ hybridization in the sulfur atoms seems necessary for the presence of the valence band near the Fermi energy, as also shown in silicon sulfide arrays [26]. 


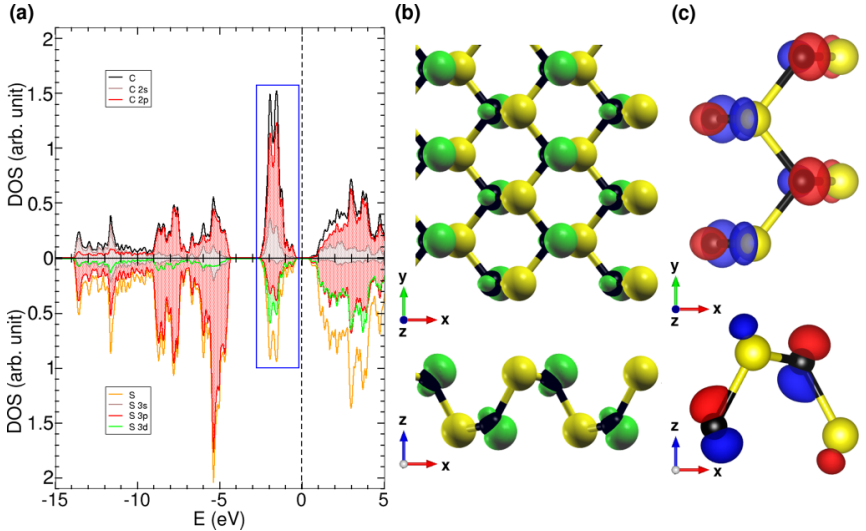

FIG. 3. Electronic properties of the ground $\alpha$ structure. (a) Density of states projected on carbon (up panel) and sulfur (down panel). (b) Spatial localized density of states for the energy peak marked up with a blue rectangle in (a). (c) Highest occupied orbital of the valence band at the $\Gamma$ point.

We then compared the carbon monosulfide and silicon monosulfide compounds [24,26], both being isoelectronic to phosphorene, in the $\alpha$ structure. Two well differentiated roles can be seen depending on the atom size, as shown in the $\alpha$ structure of Fig. 1. The smaller atom, either carbon for CS or sulfur for $\mathrm{SiS}$, is the inner atom in the structure; the larger atom is the outer one. The electronic structure produces similar trends considering that the inner atom contributes $p_{z}$ orbitals to the highest occupied orbital at the $\Gamma$ point. Figure 3(c) shows a $p_{z}$-type orbital for carbon atoms in the carbon monosulfide compounds. The main contribution to the frontier orbitals in the silicon-sulfur compound originates from the $p_{z}$ orbitals in sulfur [24]. These bands from the outer atom project a hybridized dangling bond that exhibits $p d$ hybridization for sulfur in CS, as shown by the projected density of states in Fig. 3(a), and spd-like hybridization for the frontier orbitals of silicon in SiS [24,26]. Notably, the sulfur atoms can adopt the two roles depending on the counterpart element involved in the isoelectronic compound of phosphorene.

\section{Strain-driven transition between two stable monolayer phases}

In this section we try to shed light on the striking metallization of the $\delta$ monolayer. A distortion of the $\alpha$ atomic positions could lead to the $\delta$ structure. Therefore, the monolayer could undergo a semiconductor-metal transition driven by a strain field. The $\delta$ structure is isotropic and similar to a rippled square lattice. The sulfur atoms show spd hybridization, as denoted by the projected density of states, similar to the $\alpha$ structure. A detailed analysis of the LDOS shows that the Fermi energy states are $p$ type on carbon, which can be considered as a square lattice of $p_{z}$ orbitals. Details regarding the geometry and the projected and localized density of states of the $\delta$ phase are provided in the Supplemental Material [65].

We follow the transition between the $\alpha$ and $\delta$ monolayers by changing the aspect ratio, as shown schematically in Fig. 4(a). To change the aspect ratio, we modify the unit cell shape step-by-step and transform the square cell into a rectangular cell. The unit cell during the transformation is characterized by its aspect ratio between the length and width, which is 1
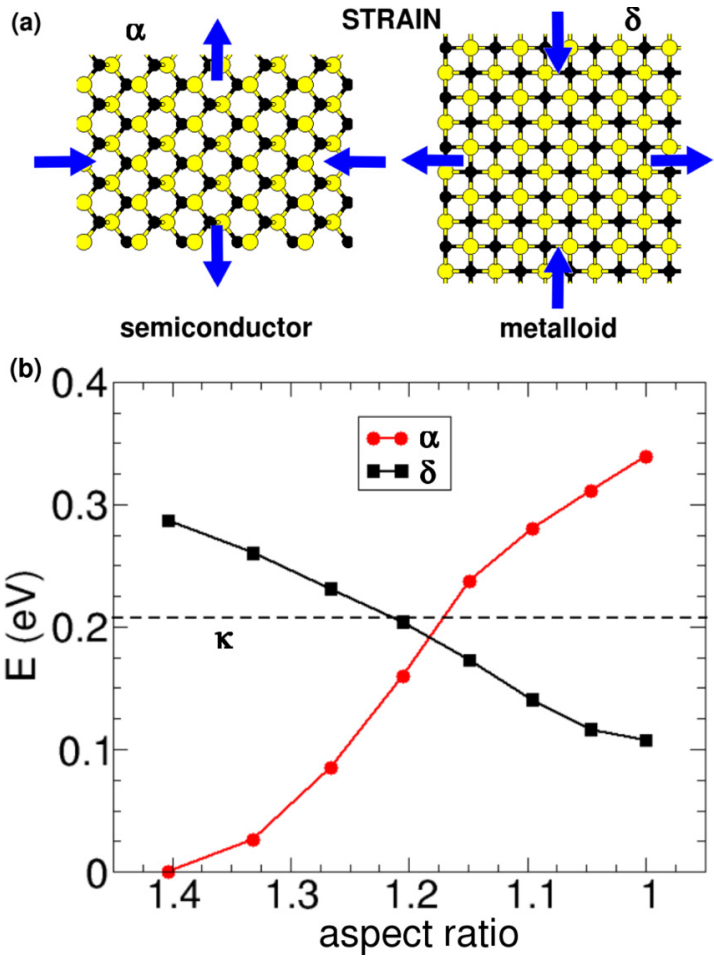

FIG. 4. Stretching the $\alpha$ and $\delta$ monolayer as shown in (a) yields an increasing energy as shown in (b), suggesting a barrier between those structures.

for the $\delta$ phase and 1.4 for the $\alpha$ phase. For each calculation, the unit cell remains fixed, and the inner atomic positions are relaxed. We expected that changing the aspect ratio of the $\delta$ monolayer would transform it into the $\alpha$ monolayer by relaxing the inner atomic positions. However, the inner atomic structure is retained when deforming the unit cell, and the energy increases continuously with the aspect ratio, as shown in Fig. 4(b). We also find a similar behavior when deforming the $\alpha$ monolayer. The two curves of the energy versus the aspect ratio intersect with a barrier between monolayers larger than $0.2 \mathrm{eV}$. This result confirms the stability of the $\delta$ structure. Note that the energy of the $\kappa$ monolayer, included in Fig. 4(b), is very close to the intersection point.

\section{B. Chain arrays}

We found another type of structure for carbon monosulfide, chain arrays. These arrays are obtained from a $\gamma \mathrm{SiS}$ monolayer [26] after substituting silicon with carbon atoms and being fully relaxed. Here a layer with carbon monosulfide chains becomes stabilized by more than half an electronvolt per atom compared to the most stable $\alpha$ monolayer. We obtained two different chain arrays with hexagonal and pentagonal motifs. Because the hexa chains are more stable than the penta chains by more than $0.27 \mathrm{eV}$ per atom, we focus on the hexa chains below. The penta chains are discussed in the Supplemental Material [65]. The structure with hexa-motif chains, shown in Fig. 5(a), is $0.785 \mathrm{eV}$ per atom more stable than the ground state $\alpha$ monolayer, which results in a large increase in its overall stability. The distance between the closest atoms of two different chains is larger than $3.8 \AA$, which indicates 

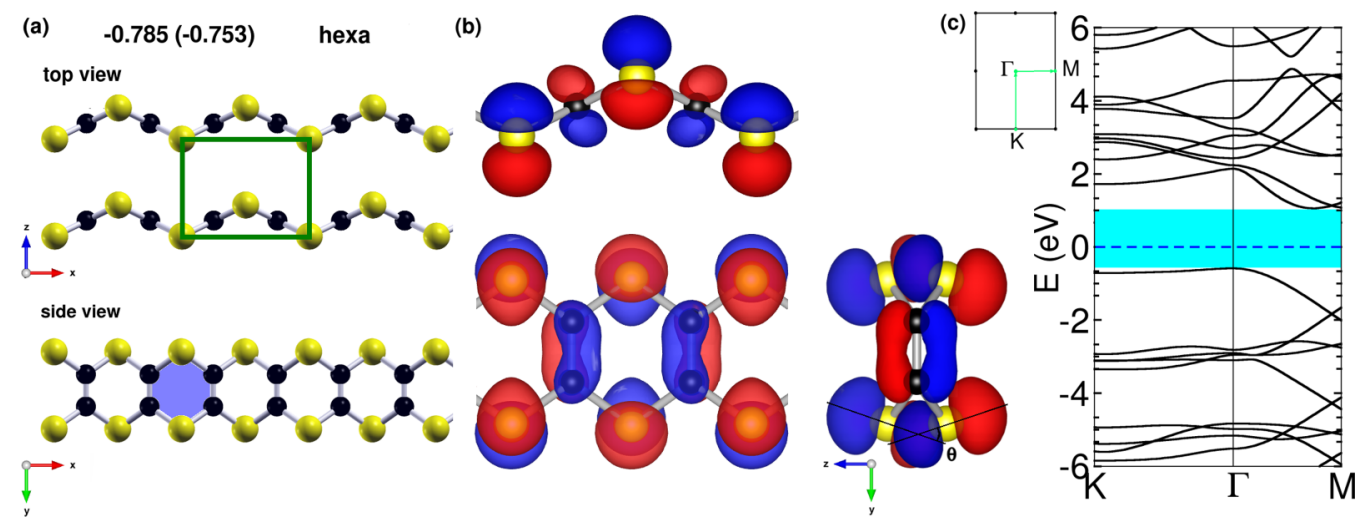

FIG. 5. (a) Geometry of the hexa chain arrays, with the hexagon motif of the structure highlighted in blue color and the unit cell marked by a green rectangle. The energies per atom obtained using SIESTA and VASP methods are given above referred to the ground state $\alpha$ monolayer. (b) Highest occupied molecular orbital (HOMO) at the $\Gamma$ point for the hexa chains. (c) Band structure of the hexa chains.

that the interactions between adjacent chains are mediated by dispersion forces. Thus, the bonds between the chains are much weaker than those established within a single chain [70].

We investigate the bonds between the atoms in the chains in more detail. Carbon atoms form covalent bonds with the sulfur atoms. Each carbon atom is bonded to two sulfur atoms with bond lengths of approximately $1.81 \AA$ and to another carbon atom with a bond length of $1.38 \AA$. The short bond lengths between the carbon atoms indicate the existence of a double bond containing a $\sigma$ and $\pi$ bond. The $\pi$ bond is spatially indicated by the highest occupied orbital at the $\Gamma$ point in Fig. 5(b), which is formed by two $p_{z}$-type orbitals from each pair of carbon atoms. The angles between the three bonds established by each carbon atom are $122^{\circ}, 122^{\circ}$, and $115^{\circ}$. The fact that the three bonds are planar and form angles close to $120^{\circ}$ is attributed to $s p^{2}$ hybridization. The remaining $p_{z}$ orbital is used to form a double $\pi$ bond with the neighboring carbon atom. Focusing now on the sulfur atoms, each atom establishes two bonds with carbon atoms. The sulfur atoms contribute with $p$ orbitals to the highest occupied orbital at the $\Gamma$ point, as shown in Fig. 5(b).

The chains adopt a zigzag pattern with kinks in the sulfur atoms, as shown in the top view of Fig. 5(a). The zigzag chains are $103 \mathrm{meV}$ per atom more stable than the straight chains with no kinks. Another kinked chain combining long and short stretches lies $20 \mathrm{meV}$ higher than the ground state of the hexa chains. Thus, the kinks stabilize carbon monosulfide chains and occur at sulfur atoms because sulfur prefers $s p^{3}$ hybridization. Note that carbon atoms remain planar with the three bonds in the same plane because carbon prefers $s p^{2}$ hybridization. However, the kinks cause sulfur $p_{z}$ orbitals to tilt with respect to $z$ axis, forming an angle $\theta$ of $40^{\circ}$ between two consecutive $p_{z}$ orbitals, as presented in Fig. 5(b).

The analysis of the projected density of states for the hexa chain array, provided in the Supplemental Material [65], shows that $p$-type contributions are dominant near the Fermi energy, in contrast to the $\alpha$ and $\delta$ monolayers. The charge reorganization under the Mulliken scheme results in a net transfer of almost 0.6 electrons from each carbon atom towards the sulfur atoms. Furthermore, we analyzed the band structure shown in Fig. 5(c) along two directions: parallel and perpendicular to the chains. The hexa chain array is a semiconductor with a band gap of $1.10 \mathrm{eV}$.

\section{FINAL REMARKS}

Finally, we comment on the overall order of stability between the different layered nanostructures of carbon monosulfide we have studied thus far. We find that chains, thin films, and monolayers sequentially increase in energy. The different energies occur due to hybridization and the number of bonds between the carbon and sulfur atoms in each structure, as summarized in Fig. 6. The $\alpha$ monolayer, taken as a reference, presents $s p^{3}$ hybridization for the carbon atoms with three bonds and a dangling bond, and $s p d$ hybridization for the sulfur atoms with three bonds. The thin films have a lower energy per atom because the carbon atoms establish a fourth bond and the sulfur atoms lose a bond. It is noteworthy

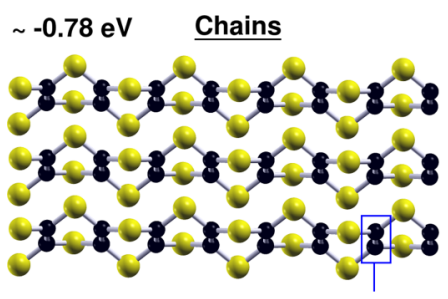

C: sp2, three bonds (1 double) S: sp3, 2 bonds

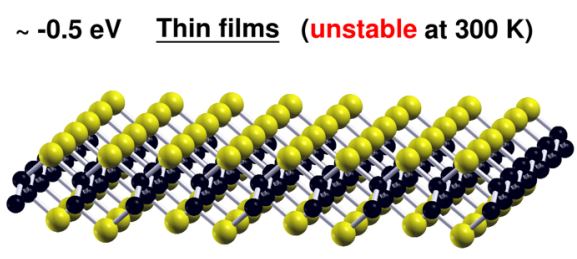

C: $\operatorname{sp} 3,4$ bonds S: sp3, 2 bonds

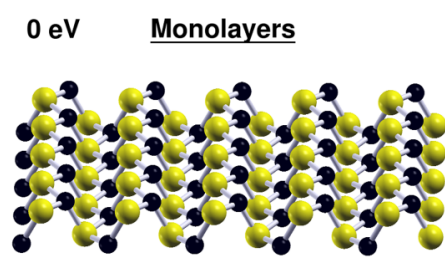

C: $\mathbf{s p 3}, 3$ bonds S: spd, 3 bonds

FIG. 6. Comparison of energetic stability, number of bonds, and hybridizations for carbon monosulfide layered nanostructures. Note that the most stable structures are the chain arrays. 
that sulfur atoms prefer to establish two bonds to fulfill the octet rule $[25,26]$. From the molecular simulations, the thin films are unstable at room temperature. The hexa chain arrays exhibit improved energy and stability with respect to the thin films. The increased stability of the chains is ascribed to the preference of carbon to undergo $s p^{2}$ hybridization with two single bonds and a double bond, as opposed to the fourfold coordination of the $s p^{3}$ hybridization in the thin films.

In summary, we presented new stable nanostructures for carbon monosulfide, which include stable chain arrays and monolayers. On the one hand, the chain arrays, which are more stable than monolayers, present hexagonal and pentagonal patterns with carbon and sulfur atoms that display $s p^{2}$ hybridization. These results ask next for the synthesis of carbon monosulfide structures as freestanding chains, but they will exhibit enhanced growth when supported on some substrates as chain arrays. On the other hand, the monolayers show $s p^{3}$ and $s p d$ hybridization in the carbon and sulfur atoms, respectively. The ground state $\alpha$ monolayer has the structure of a single layer of black phosphorus with an indirect band gap of approximately $1 \mathrm{eV}$. The strain could induce a transition between the most stable $\alpha$ and $\delta$ monolayers. Because the $\alpha$ monolayer is a semiconductor and the $\delta$ monolayer is metallic, the $\alpha$ to $\delta$ transition would enable the ability to control the conductivity by applying strain, an effect that is promising for designing new electronic devices.

\section{ACKNOWLEDGMENTS}

This work was partially supported by Projects FIS201348286-C02-01-P and FIS2016-76617-P of the Spanish Ministry of Economy and Competitiveness MINECO, the Basque Government under the ELKARTEK project (SUPER), and the University of the Basque Country (Grant No. IT-756-13). T.A.-L. acknowledges the grant of the MPC Material Physics Center - San Sebastián. F.A.-G. acknowledges the DIPC for their generous hospitality. The authors also want to acknowledge the DIPC computer center.
[1] K. S. Novoselov, A. K. Geim, S. V. Morozov, D. Jiang, Y. Zhang, S. V. Dubonos, I. V. Grigorieva, and A. A. Firsov, Science 306, 666 (2004).

[2] K. S. Novoselov, V. I. Fal'ko, L. Colombo, P. R. Gellert, M. G. Schwab, and K. Kim, Nature (London) 490, 192 (2012).

[3] X. Huang, Z. Yin, S. Wu, X. Qi, Q. He, Q. Zhang, Q. Yan, F. Boey, and H. Zhang, Small 7, 1876 (2011).

[4] A. K. Geim, Science 324, 1530 (2009).

[5] A. H. C. Neto, F. Guinea, N. M. R. Peres, K. S. Novoselov, and A. K. Geim, Rev. Mod. Phys. 81, 109 (2009).

[6] K. I. Bolotin, K. J. Sikes, Z. Jiang, M. Klima, G. Fudenberg, J. Hone, P. Kim, and H. L. Stormer, Solid State Commun. 146, 351 (2008).

[7] Y.Zhang, Y.-W. Tan, H. L. Stormer, and P. Kim, Nature (London) 438, 201 (2005).

[8] F. Schwierz, Nat. Nanotechnol. 5, 487 (2010).

[9] T. Alonso-Lanza, A. Ayuela, and F. Aguilera-Granja, Phys. Chem. Chem. Phys. 18, 21913 (2016).

[10] J. L. Lado, J. W. González, and J. Fernández-Rossier, Phys. Rev. B 88, 035448 (2013).

[11] J. W. González, H. Santos, E. Prada, L. Brey, and L. Chico, Phys. Rev. B 83, 205402 (2011).

[12] J. W. González, M. Pacheco, L. Rosales, and P. A. Orellana, Phys. Rev. B 83, 155450 (2011).

[13] C.-K. Chang, S. Kataria, C.-C. Kuo, A. Ganguly, B.-Y. Wang, J.-Y. Hwang, K.-J. Huang, W.-H. Yang, S.-B. Wang, C.-H. Chuang et al., ACS Nano 7, 1333 (2013).

[14] Z. H. Ni, T. Yu, Y. H. Lu, Y. Y. Wang, Y. P. Feng, and Z. X. Shen, ACS Nano 2, 2301 (2008).

[15] Y. Gogotsi, MRS Bull. 40, 1110 (2015).

[16] A. Hashimoto, K. Suenaga, A. Gloter, K. Urita, and S. Iijima, Nature (London) 430, 870 (2004).

[17] Z. Liu, K. Suenaga, P. J. F. Harris, and S. Iijima, Phys. Rev. Lett. 102, 015501 (2009).

[18] C. Jin, H. Lan, L. Peng, K. Suenaga, and S. Iijima, Phys. Rev. Lett. 102, 205501 (2009).
[19] P. Vogt, P. De Padova, C. Quaresima, J. Avila, E. Frantzeskakis, M. C. Asensio, A. Resta, B. Ealet, and G. Le Lay, Phys. Rev. Lett. 108, 155501 (2012).

[20] B. Lalmi, H. Oughaddou, H. Enriquez, A. Kara, S. Vizzini, B. Ealet, and B. Aufray, Appl. Phys. Lett. 97, 223109 (2010).

[21] B. Aufray, A. Kara, S. Vizzini, H. Oughaddou, C. Leandri, B. Ealet, and G. Le Lay, Appl. Phys. Lett. 96, 183102 (2010).

[22] S. Balendhran, S. Walia, H. Nili, S. Sriram, and M. Bhaskaran, Small 11, 640 (2015).

[23] A. Zunger, A. Katzir, and A. Halperin, Phys. Rev. B 13, 5560 (1976).

[24] Z. Zhu, J. Guan, D. Liu, and D. Tománek, ACS Nano 9, 8284 (2015).

[25] J.-H. Yang, Y. Zhang, W.-J. Yin, X. Gong, B. I. Yakobson, and S.-H. Wei, Nano Lett. 16, 1110 (2016).

[26] T. Alonso-Lanza, A. Ayuela, and F. Aguilera-Granja, Phys. Rev. B 94, 245441 (2016).

[27] H. Liu, A. T. Neal, Z. Zhu, Z. Luo, X. Xu, D. Tománek, and P. D. Ye, ACS Nano 8, 4033 (2014).

[28] A. Castellanos-Gómez, L. Vicarelli, E. Prada, J. O. Island, K. L. Narasimha-Acharya, S. I. Blanter, D. J. Groenendijk, M. Buscema, G. A. Steele, J. V. Alvarez et al., 2D Mater. 1, 025001 (2014).

[29] F. Xia, H. Wang, and Y. Jia, Nat. Commun. 5, 4458 (2014).

[30] J. Dai and X. C. Zeng, J. Phys. Chem. Lett. 5, 1289 (2014).

[31] S. Beniwal, J. Hooper, D. P. Miller, P. S. Costa, G. Chen, S.-Y. Liu, P. A. Dowben, E. C. H. Sykes, E. Zurek, and A. Enders, ACS Nano 11, 2486 (2017).

[32] L. C. Gomes and A. Carvalho, Phys. Rev. B 92, 085406 (2015).

[33] J. Hong and O. Delaire, arXiv:1604.07077.

[34] W. Fang, L.-C. Zhang, G. Qin, Q.-B. Yan, Q.-R. Zheng, and G. $\mathrm{Su}$, arXiv:1603.01791.

[35] L. C. Gomes, A. Carvalho, and A. H. Castro Neto, Phys. Rev. B 92, 214103 (2015).

[36] B. R. Tuttle, S. M. Alhassan, and S. T. Pantelides, Phys. Rev. B 92, 235405 (2015). 
[37] M. Mehboudi, A. M. Dorio, W. Zhu, A. van der Zande, H. O. Churchill, A. A. Pacheco-Sanjuan, E. O. Harriss, P. Kumar, and S. Barraza-Lopez, Nano Lett. 16, 1704 (2016).

[38] E. S. Reich et al., Nature (London) 506, 19 (2014).

[39] C. Kamal and M. Ezawa, Phys. Rev. B 91, 085423 (2015).

[40] S. Mardanya, V. K. Thakur, S. Bhowmick, and A. Agarwal, Phys. Rev. B 94, 035423 (2016).

[41] S. Zhang, Z. Yan, Y. Li, Z. Chen, and H. Zeng, Angew. Chem. Int. Ed. 54, 3112 (2015).

[42] W. Yu, C.-Y. Niu, Z. Zhu, X. Wang, and W.-B. Zhang, J. Mater. Chem. C 4, 6581 (2016).

[43] H. Xiao, F. Hao, X. Liao, X. Shi, Y. Zhang, and X. Chen, arXiv:1603.01957.

[44] Q. Wang, W. Yu, X. Fu, C. Qiao, C. Xia, and Y. Jia, Phys. Chem. Chem. Phys. 18, 8158 (2016).

[45] L. C. Gomes, A. Carvalho, and A. H. Castro Neto, Phys. Rev. B 94, 054103 (2016).

[46] I. S. S. de Oliveira and R. Longuinhos, Phys. Rev. B 94, 035440 (2016).

[47] Z. Ma, B. Wang, L. Ou, Y. Zhang, X. Zhang, and Z. Zhou, Nanotechnology 27, 415203 (2016).

[48] Y. Chen, Q. Sun, and P. Jena, J. Mater. Chem. C 4, 6353 (2016).

[49] F. Li, X. Liu, Y. Wang, and Y. Li, J. Mater. Chem. C 4, 2155 (2016).

[50] J. de Sousa, T. Botari, E. Perim, R. Bizao, and D. S. Galvao, RSC Adv. 6, 76915 (2016).

[51] J. Guan, D. Liu, Z. Zhu, and D. Tománek, Nano Lett. 16, 3247 (2016).

[52] G. Wang, R. Pandey, and S. P. Karna, Nanoscale 8, 8819 (2016).

[53] J. P. Perdew, K. Burke, and M. Ernzerhof, Phy. Rev. Lett. 77, 3865 (1996).

[54] N. Troullier and J. L. Martins, Phys. Rev. B 43, 1993 (1991).

[55] T. Alonso-Lanza, A. Ayuela, and F. Aguilera-Granja, Chem. Phys. Chem. 16, 3700 (2015).

[56] P. E. Blöchl, Phys. Rev. B 50, 17953 (1994).

[57] G. Kresse and D. Joubert, Phys. Rev. B 59, 1758 (1999).
[58] P. Giannozzi, S. Baroni, N. Bonini, M. Calandra, R. Car, C. Cavazzoni, D. Ceresoli, G. L. Chiarotti, M. Cococcioni, I. Dabo et al., J. Phys. Condens. Matter 21, 39 (2009).

[59] M. Dion, H. Rydberg, E. Schröder, D. C. Langreth, and B. I. Lundqvist, Phys. Rev. Lett. 92, 246401 (2004).

[60] J. Klimeš, D. R. Bowler, and A. Michaelides, Phys. Rev. B 83, 195131 (2011).

[61] G. Román-Pérez and J. M. Soler, Phys. Rev. Lett. 103, 096102 (2009).

[62] A. M. Souza, A. R. Rocha, A. Fazzio, and A. J. R. da Silva, AIP Adv. 2, 032115 (2012).

[63] Z. Zanolli and J.-C. Charlier, Phys. Rev. B 80, 155447 (2009).

[64] E. Hobi, Jr., R. B. Pontes, A. Fazzio, and A. J. R. da Silva, Phys. Rev. B 81, 201406(R) (2010).

[65] See Supplemental Material at http://link.aps.org/supplemental/ 10.1103/PhysRevMaterials.1.024001 for more details on the geometry and the electronic structure of monolayers (specifically the $\delta$ monolayer), chains (hexa and penta) and thin films, and phonon dispersion and molecular dynamics simulations.

[66] L.-C. Zhang, G. Qin, W.-Z. Fang, H.-J. Cui, Q.-R. Zheng, Q.-B. Yan, and G. Su, Sci. Rep. 6, 19830 (2016).

[67] J. L. Zhang, S. Zhao, C. Han, Z. Wang, S. Zhong, S. Sun, R. Guo, X. Zhou, C. D. Gu, K. D. Yuan et al., Nano Lett. 16, 4903 (2016).

[68] Z. Zhu and D. Tománek, Phys. Rev. Lett. 112, 176802 (2014).

[69] We integrate the charge within a sphere around the atom in graphene and in CS. Graphene has four electrons in a sphere of radius $0.96 \AA$. For $\mathrm{CS}$, the alpha and delta monolayers within the same sphere around $\mathrm{C}$ atoms have around 3.5 electrons. The carbon charge is thus moved to sulfur atoms, supporting the results obtained within the Mulliken scheme.

[70] Although the interactions between chains due to dispersion forces are beyond the actual scope of the paper, we tested the hexa chains using van der Waals interactions in the VASP method. The stability was enhanced by approximately $70 \mathrm{meV}$ per atom. 